\title{
Demography of Startup Software Companies: An Empirical Investigation on the Success and Failure
}

\author{
Kaniz Fatema \\ Professional Member \\ ACM
}

\author{
M.M. Mahbubul Syeed \\ Professional Member \\ ACM
}

\author{
M. Saef Ullah Miah \\ PhD Researcher \\ UMP, Malaysia
}

\begin{abstract}
Startup software company can be envisage as a company committed to deliver innovative solution to market with four primary objectives, namely, product, service, process and platform. Software startups are quite distinct from traditional mature software companies. These companies develop state-of-the-art, software-intensive products within limited time frames and with few resources. They also introduce new challenges relevant to software engineering research which in turn lead to ample set of empirical investigation on the topic.

This research is targeted to offer a systematic literature survey on the scientific investigations carried out on Startup software companies, specifically concentrating on their success and failure parameters and strategies to encounter symptoms of failure.
\end{abstract}

\section{Keywords}

Startup, Software Startup company, Software Development, Success, Failure, Systematic Literature Review, Investor, Management

\section{INTRODUCTION}

The term Startup was first coined in an article published by Forbes Magazine dated back in 1976 to designate a segment of software companies that are new in market, possessing a outstanding growth potential due to the technology it is developing [12] [13]. These companies have very little or no experience in operating a business and have high volatility in technologies and market [20].

With the growing need of automation incorporating high problem solving capabilities, innovative software solutions becoming an inevitable need for both the corporate users and individuals. As a consequence, new applications and cloud services targeting to resolve issues from different segments of our life are being developed [14] [15]. Most of these initiatives are brought to existence by the software startup companies. To mention a few, Facebook, UBER, Streem, Feather, Glofox have offered services that gain huge market share and credibility. However, there are numerous other cases where ingenious ideas goes in vain [16] [14].

According to statistics, Startup companies are playing a pivotal role in the economic growth of many countries [20]. For instance, in the US, every month around 476,000 new businesses are established [17], contributing to $20 \%$ of new job creation [17].
However, by its' very nature, startups possesses an evolving, unpredictable and even chaotic environment, which forces the entrepreneurs to learn, assess, comprehend and act quickly to capture a niche market for sustainability [20]. Otherwise, its very likely that the startup will fail fast. Related study reported that $75 \%$ of venture capital funded startups fail, and two-third of the startups demise within the first five years [17] [18]

Several research has been conducted to understand the underlying phenomenon of the development practices, processes, management decisions, risk factors, market volatility, and business strategies of startup companies that lead to their ultimate fate in terms of success or failure [35] [19] [36] [20] [18] [46].

These research requires a systematic characterization and reporting to get an holistic view on the issues concerning (a) what characterizes startup companies and their ecosystem, (b) what factors lead to either success or failure of an startup company, and (c) what pragmatic actions can be taken to avoid or revive from the failure?

This study therefore reporting on a systematic selection, characterization and structuring of the core literature that concern Software startup companies specially focusing on their success and failure parameters.

The paper is organized as follows, section 2 presented the research focus, research questions and a detail discussion on the review method, section 3 elaborated the findings in relation to success and failure of startup companies, and strategies to overcome the failure. Finally, threat to validity is remedied in section 4 and concluding remarks are presented in section 5

\section{REVIEW METHODOLOGY}

Systematic Literature Review (SLR) is often conducted to carry out a through and unbiased summarizing and synthesis of the existing scientific evidences on a given phenomenon in order to draw general conclusion or prelude to further research activities [1] [3]. Following the guidelines to conduct a SLR [2] [1], this research first defined a review protocol. This protocol ensures the systematic step-by-step actions taken in conducting the review, and reduces the threat to validity of the reported results. Figure 1 defines the protocol, a brief description of which is presented in this section.

\subsection{Research Objective and Research Questions}

The core focus of this literature survey is to investigate on the characteristics of the Startup companies that correlate to their success 
or failure. Therefore, 4 (four) research questions in 2 (two) broader domain of Startups' are posted. Table 1 detail the research questions along with their rationale.

\subsection{Article Selection}

As per the guideline [2] [3] [4], a concrete procedure must be defined to search, short list and select appropriate articles for conducting the review. To do so, following activities are carried out in sequence,

2.2.1 Inclusion criteria. Based on the research questions (Table 11, explicit inclusion criteria are defined. These criteria segments what articles are to be included, their quality and comprehensiveness. Following set of inclusion criteria are defined,

- Subject area of the articles must unveil strong focus on research related to startup software companies and related issues, e.g., organizational structure, process and practices, portfolio, success or failure.

-Articles published in peer reviewed journals and conferences are included for the review and excludes full books.

-All searches are based on the Title, Abstract, Keywords of the articles.

-Publication year of the articles must be between January, 2000 and December, 2018.

All the articles are assessed against these criteria through a manual analysis (discussed in Section 2.3 of title, keywords, abstract. In case of doubt conclusions are checked [2] [1] [3].

2.2.2 Search Libraries. SLR studies primarily depend on the automated keyword search of the digital libraries [4] [5]. For this study, six such libraries are searched, namely, IEEE, Springer, Google Scholar, ACM Digital Library, MDPI, and OCED. Each library is searched using the Advance Search option to maximize the inclusion of relevant articles.

2.2.3 keywords and search string. Automatic keyword search is a widely used strategy for collecting initial set of articles from digital libraries [1] [6]. For this, a set of keywords representative of the focused domain are first defined. The detail listing of keywords are presented in Table 2.2.3

To search each digital library (as listed in Section 2.2.2, search queries are formed by combining the keywords. It is to be noted that each digital library has its' own standard in defining the search queries. Therefore, while forming the search string for a digital library, corresponding guidelines are followed strictly.

Automated keyword search ended up with 376 articles. These are initial set of articles and should have false positives. Therefore, a manual screening need to be conducted to identify the correct ones.

\subsection{Manual Selection}

Automated keyword search often lists articles that are irrelevant whereas some relevant might be missing [5] [35]. Therefore, the first two authors performed a manual screening of these 376 articles by reviewing the title, keywords and abstract (and in case of doubt, checking the conclusion [2]). To reduce the researcher bias in this selection process, the third author independently examined the selected articles against the inclusion criteria (as presented in Section 2.2.1). Any disagreement is resolved through discussion. This process ended up with 48 articles.

Finally, to ensure the inclusion of other relevant but missing articles, the first author performed a non-recursive search through the references of these 48 selected articles. This process identified 3 additional articles which makes the final article count to 51 .

\subsection{Article Assessment and Synthesis}

Selected articles are assessed thoroughly by the first and third author in relation to the research questions. Findings are documented with reference to the articles. Then, these findings are scrutinized by the second author to ensure the correctness of the information. Finally, exclusive synthesis are drawn in answering the research questions. Alongside, this two pass assessment process minimizes the reviewer bias and increases the authenticity of the information [1] [3].

\section{TRANSCRIPT OF EVALUATION}

This section documents the transcript of findings in relation to the research questions. A taxonomy of the existing literature is provided to draw a holistic view on the structure and ecosystem of the startup software companies, and their success and failure parameters.

\subsection{On the Start-Up Company}

Startup software company can be envisage as a company committed to deliver innovative solution to market with four primary objectives, namely, product, service, process and platform [33].

In delivering the product, the startup working space slogan is to Done is better than perfect or Move fast and break things [20]. Therefore, startups often do rapid development of a MVP (Minimal Viable Product) to minimize time-to-market, to verify the assumptions and to capture the market share [21] [16].

Along with the product, startup founders also opting to adopt an effective and scalable business model [19]. Defining a startup business model requires two things to be established, (a) business strategy and (b) innovation model. This often depends on how the startup company captures, creates, and delivers values in economic, social, and cultural aspects of the market [34] [35].

This process of defining a business model begins with market validation. It includes three steps, e.g., problem interview, solution interview, and building a Minimum Viable Product (MVP) [42] [43]. Here, Problem interview anticipates the possible market risk and obstacle that can be faced by the company in future. Solution interview examines the startups' ability to solve a problem that it might encounter. Finally, build a MVP thick and fast to jump into the market early, and gradually mature it by attracting more investors, funds, and market share through enhanced customer satisfaction [16].

In building the $M V P$, founders have to leverage the best matched design principles to offer a coherent set of normative ideas and propositions to design [44]. However, often Startup founders need to depend on heuristics and rush into decisions that lead to catastrophic consequences [52]. Therefore, adequate mentoring and consultation with experts should provide support in taking pragmatic decisions in critical situations [44].

Engineering activities for the product development need to be tailored down to the startup context. This would allow flexibility and reactiveness within the development process and project workflow [22]. The founders will have the scope to deal with continuous unpredictability, and to establish relationship between 
cause and effect retrospect [22] [20]. They can stimulate customer feedback, evaluate alternate perspectives and solutions to act fast and opportunistically for creating customer centric value proposition and lead to a sustainable innovation [22] [60] [62].

\subsection{Start-Up Ecosystem}

A software ecosystem (SECO) is defined as "a set of actors functioning as a unit and interacting with a shared market for software and services, together with the relationships among them" [8] [9].

Based on the practice and participation of the established players in the domain of software market, researchers proposed several modeling and strategic planning methods for software ecosystem. These methods support the company stakeholders to decide on the products, services, release, market share and their frequently overlapping surrounding the software ecosystems [9] [10] [11]. Startup companies initially focus on a suitable ecosystem to adopt, run and stabilize. This ecosystem includes several independent components that should work in sync for the successful growth of the company.

The core of this ecosystem is the people who envision and invest merit and labour to translate an innovative idea into a viable business $[60]$. Translating innovative ideas into market viable inventions and products through rigorous research and development is the primary focus of these startup people [48].

The second component in the ecosystem is the organization that shapes the overall structure of the Statup company. This includes bodies, like funding and legal agencies (e.g., angel investors, start-up mentors, start-up advisors), incubators and accelerators, technical advisory and monitoring groups. Each group of organizations typically focus on specific parts of the startup at specific development stage(s) [50] |20].

Third influential factor is the professional culture within the organization. This includes but not limited to, developing the logistics and office setup, defining the office protocol and decorum, organizational hierarchy and responsibility, and marketing strategies. Having a healthy professional working culture helps employees to be creative, enhance team development, coordination and team spirit [60].

This professional culture also allows the team to be the catalyst of product development. Working on innovative product not only requires creativity, and problem solving capability, but also the ability to sustain pressure of time and resource limitations and adopt to new roles and challenges [20] [23]. Therefore, team empowerment is inevitable to boost performance and chances of success [24]. The team should learn to absorb the shock and get lesson from trial and error quickly enough to adapt to new emergent practices [25].

Additionally, people within an startup must extend their collaboration with other partners in the market through shared events, activities, locations and interactions [25]. This helps the Startup to gain market visibility, credibility and a strong hold with a strong professional network [25] [26].

Resource management is the fourth crucial factor within the startup ecosystem. Startup ecosystem provide a variety of goods and services upon which other people and companies depend on. Therefore, the principles of startup ecosystem management suggest that rather than managing individual people or organizations, resources should be managed at the level of the startup ecosystem itself [26] [22].

Resources utilized by the startups are often obtained from the stakeholders, and organizations that are an active part of the ecosystem [34]. Having a strong professional interaction with these organizations and people, the startups could have a steady flow of resources. This practice in turn will support new potential startups to emerge and at the same time strengthening the existing ones [26].

Financial and Market influence is the fifth factor that plays a central role in the startup ecosystem. Associated concerns like, financial climate, big market disruptions and big companies' transitions, control the overall structure of the ecosystem. A critical analysis and forecasting of the financial market and its' influence on the software ecosystem, often defines the course of a startup company. Therefore, the founders must learn to assess and act over the financial market as this might be the decisive factor for the their success or failure [35] [36].

Finally, the Process management, consists of all the engineering activities to carry out the product development, has a deep impact within the ecosystem. This process management should be agile, evolutionary and opportunistic [20]. Therefore, agile software development methodologies are the most viable process. Because, it allows rapid development to shorten the time-to-market, accommodate changing requirements and business strategies rather comfortably [23].

One variant of agile methodologies is the Lean Startup development [62] [21], which in addition to other agile benefits, allows to identify the most risky parts of a software business and provide a MVP to systematically test and plan modification for the next iteration.

\subsection{Success Parameters for Startup Software Companies}

Initiating a startup is a risky business due to uncertainties [27] [57]. However, studies track down several key factors that contribute to the success of Startup companies. According to BDS report (Business Dynamics Statistics, US Census Bureau, US Department of Commerce) |31| the size of startups has surged over the past decade, and average employment rate in these companies reached four-year high in 2014 with 6.1 employees per company. Average employment in firms of all ages has increased slightly during this period, from 22.4 employees per firm in 2005 to 23.5 employees per firm in 2014. Therefore, the startup companies have successfully created a large job market for skilled workers [31] [55].

Successful software startup founders should posses some distinct characteristics to translate an innovative, market viable idea into a successful product with commercial value [56]. These techno professionals are often driven by impact, resulting in passion and commitment. They are committed to stay on course and stick to the chosen path. They possesses an ideal balance between technical and business knowledge, required for management and product development [54]. They also exhibit the right mentoring relationships with employees to elevate motivation, commitment and performance. They often employ Lean Startup principles by leveraging domain specific business knowledge to raise funding in order to achieve next set of key milestones [54]. Additionally, these people often make well thought adjustment, keep their head down and have patience to adjust the mismatch between expectations and reality [55]. 
Additionally, following list of factors are positively associated with the success of Startup companies,

Speed to market: Conventional business wisdom also extends to Startup software companies as well, and that is to reach customer first. Therefore, the faster a startup can produce its service or product incorporating market demand and customer requirements, the better chance it has in capturing the market share [46].

Financial understanding: Successful management of fund is one of the key to success. Startups must plan within budget, keep themselves out of debt and grow gradually. The motto at the early stage should be to do more with less [48].

Well connected: Startup companies that invest their effort in extending market connections, gain higher degree of success. These companies should use their social network and professional communication channel to attract clients, investors, and mentors. As the old proverb goes, it's not what you know, it's who you know [57].

Quick to adapt: Successful startups are comfortable with changing needs. People in these companies can foresee the market needs and execute smart course of actions to keep-up with that [?].

Attract investors: Startup need to be smart enough to attract new investors to fund. Business leaders know how to generate capital to give their million-dollar ideas a shot [53]. Angel investors also like to take this risk with startup.

Execution: Proper and timely execution of an idea is what matters to success. Many promising ideas goes in vain just because of lack of execution. Entrepreneurs must gain business experience, expertise and leadership to lead the team in performing right thing at right time [56].

Perseverance: Startups must continue obstinately in a course of action in spite of hardship or interim failure. Management must analyse the current situation, assess possible bail outs and act fast in executing the action [54]. The majority of startups bail when money is tight or disagreements arise between founders.

\subsection{On the Failure of Startup Software Companies}

According to statistics, $75 \%$ of venture capital funded startups fail, and $60 \%$ of the startups demise within the first five years of their commencement [?] [18|. Several research has been conducted to empirically investigate on the cause of failure of these startup software companies, an exclusive summary of which is drawn bellow. Startups by its very nature trying to offer innovative solution to the market without the proof of functionality in the real world. This lack of concept validation increases the chance of failure [14]. According to [24], most of the startups fail for lack of customer validation than lack of technology.

Additionally, many entrepreneurs start without a market viability survey and a concrete plan of execution. They either just jump into it or have a very brief sketch of what to do. They often make good progress in the early stages because the milestones are close together and relatively simple to achieve, however, soon fall a part as the milestones get farther, and complex to achieve [59| |61]. Things even get more acute when hard works goes in vain due to unclear goal, vision and unprofessional execution plan [49].
There are several other factors that contribute to the failure of startup companies. For instance, lack of consistent strategies to understand the problem to be solved, and deviate vastly in the wrong directions [14]; little or no operating experience; finite resources and no control over expenditure; multiple influences, dynamic technologies and market uncertainties [48] [50].

Following is a classification of the key factors according to research that contribute to the failure of startup companies.

Not the right team formation often relates to lack of motivation, expertise, common vision and experience. Management fails in organizing the team with adequate effective people with explicit responsibilities. May be due to budget constrains, startups hire clever but inexperienced people during their early stages. These people often deliver a product that does not meet customer requirements and even unreliable and fails frequently [49].

As the project progresses and the company starting to evolve, new people with specific skill sets and experience are recruited. Due to their higher qualifications, management often put them in the driving seat with the existing members to work under them [46]. This brings significant resistance from the older members, who believes themselves to deserve special treatment which is by the way not justified with their experience and skills [14] [59].

Additionally, management many a times fail to realize the importance of developing skilled manpower over the time to carrying out distinct responsibilities of product development. Rather, a handful number of highly skilled individuals run the full show. This creates monopoly and bottleneck in all activities, which in turn often put the development process over burdened and halted [60].

Product without a business model refers to the situation where the management fail to establish objectives and goals for product development [14]. The management and stakeholders make ad-hoc decisions in many key issues, over shadowing the greater strategic plan. Such disjoint project management leads to critical resource allocation (e.g., money, manpower, logistics) on the wrong things and at the wrong time, producing very little or no return [60].

With the gradual growth of the company, new products need to be introduced. Such development must be carried out under a detailed business plan envisioned the future of the company. Teams must coordinate their activities in parallel ( e.g., product development, professional services, support, sales and marketing) under the supervision of the management. However, due to lack of proper business plan and its execution, substandard products are developed with little value to the customers [55] [56].

Lack of Project management leaves the product orphan. Due to naive management skills, no one in the organization knows who has the authority to decide on products features, development, release planning and related issues. They do not realize the importance of selecting contemporary and cutting edge technologies and components (e.g., hardware and software). This left the technology selection on the developers which lead to long term consequences [30]. For instance, developers' fail to build skills and competencies around stable platforms, which severely affect the development of reusable components, and the plan of developing a family of products to capture different market segments [29]. Additionally, recruitment of new executives in the management may ignite conflicts of interests. This affect effective leadership to lead the company towards success [27].

Product fails to meet market demand is the most cited reason why more than half of the startups fail. Failure is evident while 
startups are solving problems that are interesting to solve rather than those that has market demand [30].

Also, developed product may face challenging bugs to be resolved which keep postponing the release of a stable version as per customer demand $[20]$. This hampers the reliability of the product and in consequence, gradually loose the market share to its competitors. On the other hand, developed software might start getting overwhelming demand for new features and to make the product available on more platforms. The company also make promises to deliver the same to its customers. However, due to lack of proper management, development process get overburdened and timescales are not met $\mid 20]$. Stakeholders knew that they are investing on the product enhancement, but find little progress at the end. Management get frustrated due to not finding pragmatic solutions to speed up the process [37].

Running out of cash is an issue that contributes heavily towards failure [25]. Under funding or reckless expenditure of money, both are detrimental to survival.

Get out-competed from the market by the competitors on the ground of motivation, expertise, funding and other skills [24]. Even though such competition is not expected or even common in the domain of startup companies, still in reality many entrepreneurs jumps into the business while an idea gets hot and achieves market validation [27]. Having strong competitor(s) in the market, ignoring them all together without any viable plan to encounter, would lead to the ultimate failure and demise of the company.

\subsection{Failure Remedy Strategies}

Several key strategies are prescribed in the literature in relation to process, practices and selection of alternatives to minimize the chance of failure. Following is a summary of those proposals.

Process management practices: All engineering activities that lead to product development and management should be flexible enough to accommodate frequent changes and adopt to the market demand [15]. Therefore, agile methodologies are considered the most viable process for startups. All variants of agile practices support short iteration of development, frequent release at the end of every iteration (typically takes one or two weeks), and incremental approach to deliver features [67]. These practices facilitates the startup with fast deployment of the product, addressing changing needs of the customers and market uncertainties [68] [21].

Among the agile methodologies, XP (Extreme Programming) is the most commonly used methodology within the startups, due to its' reduced process costs and low documentation requirements [69]. Alternatively, Lean development practice can be used [21] [62|. It supports identification of risky parts of a software business, rapid prototyping with MVP for testing and reducing time to market [16].

Managerial and organizational practices: A startup should be lead by an entrepreneur who preferably have experience and expertise working on similar business domain and can offer leadership [64]. He should have the ability to adopt in dynamic environment, and can manage, motivate and guide the team to cope with fast changing needs, challenges and resource constraints $|65|$. The organizational structure and practice should offer a flexible orientation to reduce bureaucracy in decision making and approval process for rapid progress [52]. To accommodate such flexibility, team empowerment is a must. The entrepreneur or the management must train the team to learn from trial and error quickly enough to adapt to new emergent practices [66], enhance performance [66], optimize resource utilization, and adopt efficient development process to effectively deliver to customers' demand [65].

Tools and technology selection: As discussed earlier, startups often prefer to go for easy to implement tools and open source components for faster development and flatter learning curve. These choices also underpinned by the low maintenance cost which is often a big concern during the early phase of the company [63]. However, cutting edge development tools and techniques supporting new languages, objects and distributed technologies, versioning, are the best suited for the development of innovative products [15]. It supports adopting to the fast changing nature of the tech world. It may cause a spike in terms of cost, learning and adoption during the early stages, but once adopted, will support long term sustainability of company [19].

\section{VALIDITY THREAT}

Carrying out a SLR is mostly a manual task and subject to researcher bias [2]. To minimize this, a well documented approach following the suggestion of Kitchenham [1] was defined in advance and executed over the review process. Validity measures that are ensured within the definition of the review process (as presented in $\mathrm{Sec} 2$ is discussed bellow,

\subsection{Article Selection}

According to the guideline of Kitchenham [3], the inclusion criteria is set at the time of defining the review process, and the criteria are based on the research questions. This reduces the likelihood of biasing the article selection process. Articles satisfying the criterion are considered only. Additionally, manual assessment and reference checking of every selected article ensures the comprehensiveness of the review.

\subsection{Article Assessment}

Interpretation of the scholarly content of the articles are also subject to reviewer bias. Therefore, this review is conducted in a two pass method where two distinct panel of reviewers are formed to cross validate the review results (as presented in Section 2.4.

\section{CONCLUSION}

With their high problem solving capability, technical expertise, courage and enthusiasm, Startup software companies are delivering the solutions for corporate and commercial users. In the process, they contribute heavily in the economy with new jobs and GDP growth. However, such companies are often operated by people with very little or no experience in business operation, and have high volatility in technologies and markets. While some startups took very pragmatic course of actions that lead to proliferation, others fail to survive (around 60\% of all startup initiatives fails).

Several research has been conducted to understand the underlying phenomenon of the development practices, process, management, decisions of startup companies that lead to their ultimate fate in terms of success or failure. These research requires a systematic characterization and reporting to get an holistic view on the issues, like (a) what leads to either success or failure of an startup company, and (b) what pragmatic actions can be taken to avoid or revive from the failure? 
This paper reports the same through a systematic literature review. The empirically validated guidelines would support future startup initiatives to decide on critical issues that may otherwise overlooked. For instance, early recognition and management of the issues as classified in this paper along with the recovery guide can increase the chances of success for a software startup company.

\section{REFERENCES}

[1] Kitchenham B. A., Pretorius R., Budgen D., Brereton O. P., Turner M., Niazi M. and Linkman S. 2010. Systematic literature reviews in software engineering- a tertiary study, IST, vol. 52 , no. 8, pp. 792805 .

[2] Mahbubul Syeed M. M., Hammouda I. and Systa T. 2013. Evolution of Open Source Software Projects: A Systematic Literature Review, Journal of Software, Vol.8, No.11.

[3] Kitchenham B. and Charters S. 2007. Guidelines for performing systematic literature reviews in software. Engineering Technical Report EBSE-2007-01.

[4] Petticrew M. and Roberts H. 2005. Systematic reviews in the social sciences: A practical guide. Blackwell Publishing.

[5] Cornelissen B. , Zaidman A., Deursen A., Moonen L. and Koschke R. 2009. A systematic survey of program comprehension through dynamic analysis. TSE, vol. 35 , no. 5, pp. $684-702$.

[6] Catal C. and Diri B. 2009. A systematic review of software fault prediction studies. Expert Systems with Applications, vol. 36, no. 4, pp. 73467354.

[7] mite D., Wohlin C., Gorschek T., and Feldt R. 2010. Empirical evidence in global software engineering: a systematic review. ESE, vol. 15, no. 1, pp. 91118.

[8] Jansen s., Finkelstein A., and Brinkkemper s. 2009. A sense of community: A research agenda for software ecosystems. International Conference on Software Engineering.

[9] Vasilis Boucharas, Slinger Jansen, and Sjaak Brinkkemper. 2009. Formalizing software ecosystem modeling. In Proceedings of the 1st international workshop on Open component ecosystems (IWOCE 09). Association for Computing Machinery, New York, NY, USA, 4150.

[10] Jansen S., Brinkkemper S., Hunink I., and Demir C. 2008. Pragmatic and opportunistic reuse in innovative start-up companies. Software, IEEE, 25(6):4249.

[11] Brinkkemper S., van Soest I. and Jansen S. 2007. Modeling of Product Software Businesses: Investigation into Industry Product and Channel Typologies, pages 119.

[12] Smagalla, D. 2004. The truth about software startups. MIT Sloan Manage. Rev. (USA) 45(2).

[13] Blank, S. 2005. The four steps to the epiphany, 1st edn. CafePress.

[14] Crowne, M. 2002. Why software product startups fail and what to do about it. In: Proceedings International Engineering Management Conference (IEMC), pp. 338343.

[15] Sutton, S.M. 2000. The role of process in software start-up. IEEE Software 17(4), Pages 3339.

[16] Giardino C., Wang X., Abrahamsson P. 2014. Why EarlyStage Software Startups Fail: A Behavioral Framework. In: Lassenius C., Smolander K. (eds) Software Business. Towards Continuous Value Delivery. ICSOB 2014. Lecture Notes in Business Information Processing, vol 182. Springer.
[17] Fairlie R. W. 2014. Kauffman Index of Entrepreneurial Activity, Kauffman Foundation.

[18] Nobel C. 2011. Why Companies Fail - and How Their Founders Can Bounce Back. Harvard Business School.

[19] Paternoster N., Giardino C., Unterkalmsteiner M., Gorschek T. and Abrahamsson P. 2014. Software development in startup companies: A systematic mapping study, Inf. Softw. Technol.

[20] Giardino C., Unterkalmsteiner M., Paternoster N., Gorschek T. and Abrahamsson P. 2014. What Do We Know about Software Development in Startups?. in IEEE Software, vol. 31, no. 5, pp. 28-32.

[21] Ries, E. 2011. The Lean Startup: How Todays Entrepreneurs Use Continuous Innovation to Create Radically Successful Businesses. Crown Business.

[22] Kurtz C. F. and Snowden D. J. 2003. The new dynamics of strategy: Sense-making in a complex and complicated world. IBM Syst. J., vol. 42, no. 3, pp. 462483.

[23] Yu Y.-W., Chang Y.-S., Chen Y.-F. and Chu L.-S. 2012. Entrepreneurial success for high-tech start-ups - Case study of Taiwan high-tech companies, in Proceedings - 6th International Conference on Innovative Mobile and Internet Services in Ubiquitous Computing. pp. 933937.

[24] Carmel E. 1994. Time-to-completion in software package startups. Proc. 27th Hawaii Int. Conf. Syst. Sci. HICSS, pp. 498507.

[25] Sutton S. M. 2000. The role of process in software start-up. IEEE Softw., vol. 17, no. 4, pp. 3339.

[26] Jalali I. 2016. Connecting Asia's startup ecosystem, Article, in Tech in Asia.

[27] Krishna A., Agrawal A. and Choudhary A. 2016. Predicting the Outcome of Startups: Less Failure, More Success. IEEE 16th International Conference on Data Mining Workshops.

[28] Coleman G. and OConnor R. 2008. An investigation into software development process formation in software start-ups. J. Enterp. Inf. Manag., vol. 21, no. 6, pp. 633648.

[29] Brooks F. 1995. The Mythical Man Month, Addison Wesley, 2nd Ed, pp.255-257.

[30] McGrath M. 2000. Product Strategy for High Technology Companies, McGraw-Hill Education, 2nd Edition, pp.53-89.

[31] US Census Bureau. 2014. US Department of Commerce, Business Dynamics Statistics. https://www.census.gov/ces/dataproducts/bds.

[32] Stanley M. S. and EC Cubed Jr. 2000. The role of process in a software Start-Up.

[33] Robehmed N. 2014. What Is A Startup?, Business Insider.

[34] Stephen D.H.T. and Tzu-Tang Lan. 1984. Development of a Startup Business - A Complexity, In Theory Perspective - Research Gate.

[35] Abrar A.S. and Maheshwari S. 1998. Entrepreneurial Characteristics and Strategic Choices of New Venture Firms, Twelfth AIMS International Conference on Management.

[36] Sauka A. and Chepurenko A. 2017. Entrepreneurship in Transition Economies Diversity, Trends, and Perspectives, OCED.

[37] Thomas, E. B. 2000. The failure of success: challenges of disseminating effective substance abuse preventions programs, Journal of Community Psychology.

[38] Fayad M.E., Laitinen M. and Robert P. Ward. 2000. Software Engineering in the Small, Communications of the ACM. 
[39] Karlheinz K. 2001. Knowledge, Learning and IT support in a small software company, Journal of Knowledge Management.

[40] Chau T. and Frank Maurer F. 2001. A Case Study of Wikibased Experience Repository at a Medium-sized Software Company, Proceedings of the 3rd international conference on Knowledge capture.

[41] Crowne M. 2002. Why software product startups fail and what to do about it Evolution of software product development in startup companies, Proceedings of The International Engineering.

[42] Schick H., Marxen S. and Freiman J. 2002. Sustainability Issues for Start-up Entrepreneurs, Greener Management International.

[43] Himola O. P., Helo P., Ojala L. 2003. The Value of Product Development Lead Time in Software Startup, System Dynamics Review.

[44] Paternoster N., Giardino C., Unterkalmsteiner M. and Gorschek T. 2014. Supplementary material to Software development in startup companies: A systematic mapping study, In International Conference of Software Business, pp. 27-41.

[45] Boehm B., Guo L., Huang. 2003. Value-Based Software Engineering: A Case Study, IEEE Computer, 36 (3).

[46] Gelderen M.V., Thurik R., Bosma N. 2005. Success and Risk Factors in the Pre-Startup Phase, Small Business Economics, 24(4), pp 365-380.

[47] Martin D., Rooksby J., Rouncefield M. and Sommerville I. 2007. Good Organisational Reasons for Bad Software Testing: An Ethnographic Study of Testing in a Small Software Company, ICSE Proceedings of the 29th international conference on Software Engineering, pp 602-611.

[48] Oktaba P., Piattini M. 2008. Software Process Improvement for Small and Medium Enterprises: Techniques and Case Studies, ACM Digital Library.

[49] Nann S., Krauss J., Schober M., Peter A. Gloor, Fischbacha K. and Hauke Fuhr. The Power of Alumni Networks -Success of Startup Companies Correlates With Online Social Network Structure of Its Founders, MIT Sloan School Working Paper 4766-10.

[50] Barbosa O., Alves C. 2011. A Systematic Mapping Study on Software Ecosystem, Proceedings of the Workshop on Software Ecosystems.

[51] Swamidass P.M. 2012. University startups as a commercialization alternative: lessons from three contrasting case studies, The Journal of Technology Transfer.

[52] Bosch J., Olsson H.H., Bjork J. and Ljungblad J. 2013. The Early Stage Software Startup Development Model: A Framework for Operationalizing Lean Principles in Software Startups, LESS, LNBIP 167, pp. 1-15.

[53] Marciuska S., Gencel C. and Abrahamsson P. 2013. Exploring How Feature Usage Relates to Customer Perceived Value: A Case Study in a Startup Company, ICSOB, LNBIP 150, PP.166-177.

[54] Abelein H., Sharp H. and Paech B. 2013. Does Involving Users in Software Development Really Influence System Success?, IEEE Software, 30(6).

[55] Mason C., Brown R. 2014. Entrepreneurial ecosystems and growth oriented entrepreneurship, OCED.

[56] Fagerholm F., Paasivaara M., Jedlitschka A., Kuvaja P., Kuhrmann M., Tomi $\mathrm{M}$ annist .o, J urgen $\mathrm{M}$ uch, and
Raatikainen M. 2014. Product-Focused Software Process Improvement, 15th International Conference, PROFES Helsinki, Finland.

[57] Verbovskii V.A. 2014. Basics of successful startup development in the field of innovation, Journal of Economics and Social Sciences.

[58] Klotins E., Unterkalmstenier M. and Gorschek T. 2014. Software Engineering Knowledge Areas in Startup Companies: a mapping study, Blekinge Institute of Technology.

[59] Lose T., Tengeh R.K. 2015. The Sustainability and Challenges of Business Incubators in the Western Cape Province, South Africa, Sustainability, pp 14344-14357.

[60] Unterkalmsteinera M., Abrahamssonb P., XiaoFeng Wangc, Anh Nguyen-Duca, Syed Shahd, Sohaib Shahid Bajwac, Guido H. Baltese, Kieran Conboyf, Eoin Cullinaf, Denis Dennehyf, Edisonc H. and others. 2016. Software Startups A Research Agenda, e-Informatica Software Engineering Journal, 10(1), pp: 89123.

[61] Nobel, C. 2011. Why companies fail and how their founders can bounce back. Working knowledge.

[62] Rainer H. 2015. Self-regulated learning, team learning and project performance in entrepreneurship education: Learning in a lean startup environment, Technological Forecasting and Social Change. 100: 2128.

[63] Kuhn D. 1989. Selecting and effectively using a computer aided software engineering tool, in: Annual Westinghouse Computer Symposium.

[64] Kakati M. 2003. Success criteria in high-tech new ventures, Technovation, 23(5), pp 447457.

[65] Yoo C., Yang D., Kim H. and Heo E. 2012. Key Value Drivers of Startup Companies in the New Media Industry-The Case of Online Games in Korea, Journal of Media Economics 25(4), pp 244260.

[66] Midler C. and Silberzahn P. 2008. Managing robust development process for high-tech startups through multi-project learning: The case of two European start-ups, International Journal of Project Management, 26(5), pp 479486.

[67] Taipale M. 2010. Huitale - A story of a Finnish lean startup, Lean Enterprise Software and Systems, Vol. 65 of Lecture Notes in Business Information Processing, pp. 111114.

[68] da Silva A. and Kon F. 2005. Xp south of the equator: An experience implementing XP in Brazil, Extreme Programming and Agile Processes, pp 1018.

[69] Coleman G. and OConnor R. 2007. Using grounded theory to understand software process improvement: A study of Irish software product companies, Information and Software Technology 49(6), pp 654667. 


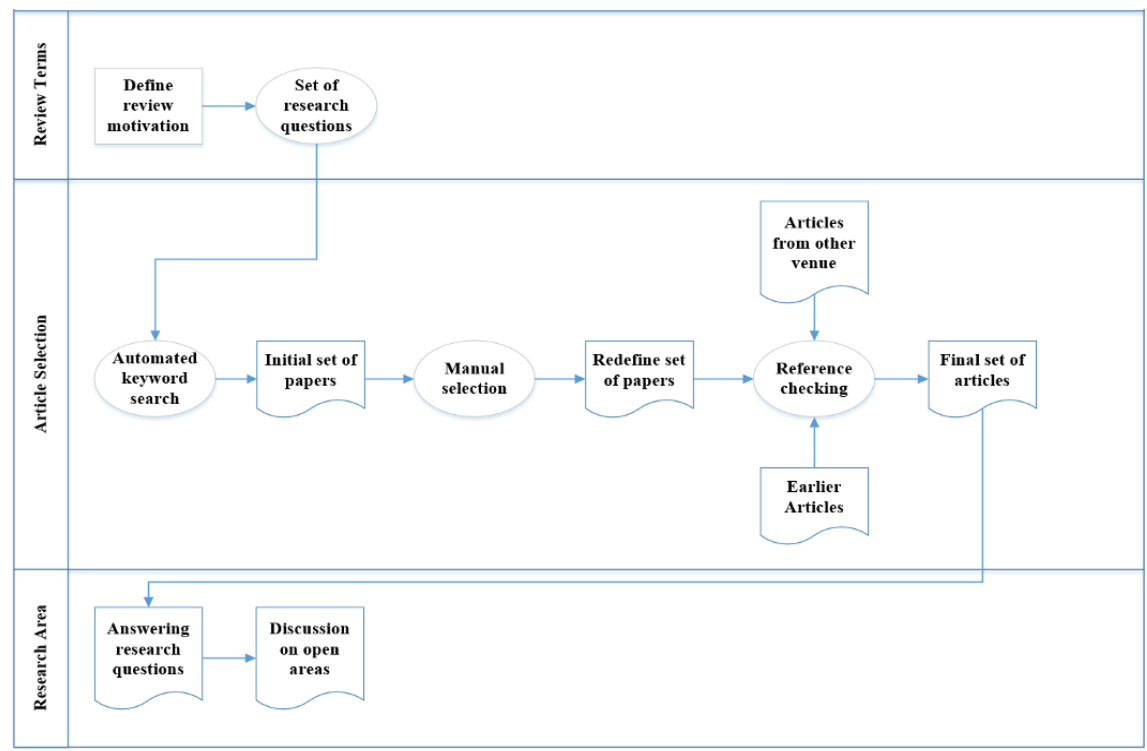

Fig. 1. Brief sketch of the review protocol followed

Table 1. Research Questions

\begin{tabular}{||lll||}
\hline Start-up Domain & Question & Motivation \\
\hline \hline Organigram and Practice & $\begin{array}{l}\text { 1. What is a start-up or startup company? } \\
\text { 2. What is a startup ecosystem? }\end{array}$ & $\begin{array}{l}\text { To define what constitutes a startup software paradigm. } \\
\text { To define the key properties of startup environment, actors and } \\
\text { factors within the ecosystem to consider. }\end{array}$ \\
\hline Portfolio & $\begin{array}{l}\text { 3. What are the success parameters for a startup company? } \\
\text { 4.What are the reasons a startup company fails and what can } \\
\text { be done for the avoidance? }\end{array}$ & $\begin{array}{l}\text { To determine the factors influencing growth and success of the } \\
\text { statrup companies. } \\
\text { To trace the reason of failure and strategies to remedy or re- } \\
\text { cover from that. }\end{array}$ \\
\hline
\end{tabular}

Table 2. Keywords for Digital Library Search

\begin{tabular}{||l|l||}
\hline Startup & newly emerged, fast-growing business, innovative product, service, process, platform, effective \\
\hline Software Startup & software startup, software start-up, early-stage firm, early-stage company, startup company, lean startup \\
\hline Software Development & develop, engineering, engineer, model, construct, implement, code, create, build \\
\hline Strategy & $\begin{array}{l}\text { product, service, process, methodology, tool, method, practice, artifact, artefact, quality, software, move, } \\
\text { action, management }\end{array}$ \\
\hline Software Company & software, technology, distribution, software product development \\
\hline Mapping & data mapping, overview, upscale, connection \\
\hline Investor & angel, angel-investor, venture capital, funding, business-angel, venture companies \\
\hline Management & managing, administration, organization, business, corporation, strategy, coordinate \\
\hline
\end{tabular}

